\title{
Análisis económico para el diseño de una agencia de viajes Vital Tur
}

\section{Analysis economical financial for the design of a travel agency Vital Tur}

1 Adlyn Pereira González

Universidad de La Habana, Facultad de Turismo, Carrera de Licenciatura en Turismo,

Cuba.

yumayry@nauta.cu

2 Thalia Lee Pons

Universidad de La Habana, Facultad de Turismo, Carrera de Licenciatura en Turismo,

Cuba.

thalialeepons@gmail.com

3 Roberto Modesto Llanes

Universidad de La Habana, Facultad de Turismo, Departamento de Turismo, Cuba.

llanes@ftur.uh.cu

4 Yolanda Tatiana Carrasco Ruano

Visionario Digital, Ambato, Ecuador,

https://orcid.org/0000-0002-5294-8202

tatianacarrasco@cienciadigital.org

Artículo de Investigación Científica y Tecnológica

Enviado: $16 / 12 / 2021$

Revisado: 30/12/2021

Aceptado: 05/01/2022

Publicado:26/02/2022

DOI: https://doi.org/10.33262/concienciadigital.v5i1.2079

Cítese: $\quad$ Pereira González, A., Lee Pons, T., Modesto Llanes, R., \& Carrasco Ruano, Y. T. (2022). Análisis económico para el diseño de una agencia de viajes Vital Tur . ConcienciaDigital, 5(1), 222-237. https://doi.org/10.33262/concienciadigital.v5i1.2079

CONCIENCIA DIGITAL, es una Revista Multidisciplinar, Trimestral, que se publicará en soporte electrónico tiene como misión contribuir a la formación de profesionales competentes con visión humanística y crítica que sean capaces de exponer sus resultados investigativos y científicos en la misma medida que se promueva mediante su intervención cambios positivos en la sociedad. https://concienciadigital.org La revista es editada por la Editorial Ciencia Digital (Editorial de prestigio registrada en la Cámara Ecuatoriana de Libro con No de Afiliación 663) www.celibro.org.ec 


\section{Palabras} claves:

envejecimiento, agencia de viajes, análisis, económico, financiero

\section{Keywords:}

ageing, travel agency, analysis, economic, financial.

\begin{abstract}
El envejecimiento progresivo es una realidad mundial, gracias al aumento de la esperanza de vida. La denominada tercera edad constituye uno de los segmentos de mercado más atractivos para el sector turístico. Este grupo, cada vez más numeroso, cuyos individuos están en plenitud de facultades y con una disponibilidad adquisitiva considerable, poseen cierta potencialidad, respaldada también por un mayor tiempo libre. A pesar de la gran oportunidad que representa el segmento, se evidencia cierta escasez en cuanto a ofertas orientadas a dicho segmento. El segmento senior está actualmente cada vez más vinculado a la utilización de su tiempo libre en actividades turísticas que le proporcionen enriquecimiento y experiencia, de ahí que las tendencias a viajar y conocer nuevas culturas y destinos es una motivación elevada. El presente informe, tratará sobre la elaboración de un proyecto de agencia de viajes, especializada en satisfacer las necesidades del turista de la tercera edad, utilizando el análisis económico financiero como método matemático.
\end{abstract}

\section{Abstract}

Progressive ageing is a worldwide reality, due to the increase in life expectancy. The named third age (seniors) are one of the most attractive niche markets to the tourism sector. This niche, becoming ever more numerous, whose individuals are in plenty of faculties and has a considerable purchasing level, own a certain potentiality, also supported by a high amount of free time. Notwithstanding the fantastic opportunity that de niche represents, it is seen certain lack of service offers focused to this niche. The senior niche is now a days ever more linked to enjoy their free time in touristic activities that can provide them spiritual enrichment and experiences, which is why the growing trend of traveling and the interests in new cultures and destinies. The present report develops a project of a Travel Agency specialized on meeting the requirements of senior tourists, using the economic and financial analysis as mathematical method.

\section{Introducción}

El turismo en el último siglo ha experimentado un desarrollo continuo y vertiginoso, llevado de la mano de los constantes avances científicos-técnicos y tecnológicos, lo que 
ha propiciado el aumento constante de los viajes, perfeccionándose los medios de transporte tanto aéreos, terrestres, como marítimos, originando la aparición de entidades que actúen de intermediarias entre los clientes y los prestatarios, aglutinando una serie de productos y servicios, que son las agencias de viajes (Laborde, 2009).

A partir de la década de los sesenta, en el siglo XX, sobre todo en los países desarrollados, las Agencias de Viajes (AAVV) desempeñaron un papel fundamental en la expansión de las corrientes turísticas, y en el advenimiento del turismo de masas, llegando a manejar grandes volúmenes de pasajeros y orientando el crecimiento del turismo hacia determinados destinos, bajo esquemas de venta de servicios integrados a un solo precio (Pomeda, 2001), ocupando un sitio estratégico en el desarrollo y explotación racionales del sector turístico, todo ello derivado de su labor en la comercialización del producto turístico.

El envejecimiento progresivo ha generado la curiosidad de muchos. Según la Organización Mundial de la Salud (OMS, 2021), se estima que entre 2000 y 2050 la población mayor de 60 años se duplicará, es decir que, pasará del 11\% al 22\%; en números absolutos pasará de 605 millones a 2.000 millones, en el transcurso de medio siglo. Esto se debe al aumento de la esperanza de vida en comparación con años atrás.

La población mayor representa un mercado potencial para el turismo, por lo que ha despertado interés, y se está tratando de profundizar en este perfil mediante investigaciones por parte de actores y gestores involucrados en el sector, como puede ser la Organización Mundial del Turismo (OMT, 2016), la cual se compromete a impulsar la accesibilidad para este segmento, tal como se discutió con motivo de Día Mundial del Turismo, en el año 2016.

Cuba por su riqueza natural, imperecedero valor cultural y patrimonio histórico, sería el lugar propicio para explotar la potencialidad del turista senior o segmento de la tercera edad. Sin embargo, no abunda en el destino, ofertas turísticas encaminadas a satisfacer específicamente las necesidades de dicho segmento, el cual goza de una atractividad para aumentar el flujo turístico en el destino, precisamente por contar con más tiempo y recursos que un turista convencional.

Si al análisis de las oportunidades del sector turístico dentro del mercado de la tercera edad se le incluye un análisis económico- financiero detallado respecto a las proyecciones de estabilidad financiera y su capacidad productiva desde el punto de vista económico, el proyecto de creación de una agencia de viajes especializada adquiere mayor solidez y efectividad de implementación. De ahí la importancia de la utilización de métodos matemáticos a la hora de creación de proyectos turísticos. 
Por tanto, se formula como objetivo general de la investigación diseñar el proyecto de creación de la agencia de viajes especializada VitalTur, en el destino Cuba.

\section{Metodología}

Toda empresa determina su posición a partir de dos situaciones fundamentales: la situación económica y la situación financiera. De tal forma, mientras que la situación económica define la capacidad de la empresa para generar beneficios, la situación financiera se refiere a sus habilidades de solvencia y liquidez.

Un análisis económico independiente evalúa la capacidad productora de la instalación en diferentes periodos, evaluando el funcionamiento productivo de la misma. Por su parte, el análisis financiero se enfoca en el control del uso adecuado de los recursos financieros y su aprovechamiento para incrementar el poder inversionista.

A la hora de un estudio del comportamiento general de las empresas, en función de estructurar un esquema sistémico de correlación entre sus aspectos contables, que convergen en el camino de perfeccionamiento y mejora de los procedimientos internos y actividades externas de las instalaciones, se utiliza un método combinado conocido como Análisis Económico- Financiero; el cual fue utilizado en el proyecto de creación de la agencia de viajes VitalTur.

El análisis económico-financiero, también conocido como análisis de estados financieros, análisis de balances o análisis contable, es un conjunto de técnicas para diagnosticar la situación de la empresa, detectar reservas y tomar las decisiones adecuadas. Su utilidad está en función del objetivo que se defina en el estudio y de la posición de quien lo realiza: desde una perspectiva interna, la dirección de la empresa puede tomar decisiones que corrijan los puntos débiles que puedan amenazar el futuro, así como potenciar los puntos fuertes para alcanzar los objetivos (Nogueira-Rivera et al., 2017).

Generalizando un poco las palabras anteriores, los métodos de análisis económicofinanciero proporcionan un diagnóstico de la situación de la empresa, y basado en su anticipación interpretativa de la información contable, evalúa los hechos económicos y confecciona una base sólida para la proyección de estrategias futuras.

En sus primeras aplicaciones, surge como ciencia de balance, limitándose al análisis del balance y la situación financiera. A medida que fueron evolucionando las tendencias administrativas y el uso de las tecnologías, estos se fueron perfeccionando en sus procesos de análisis, interpretación y retroalimentación.

Hoy en día este análisis ofrece ventajas significativas dentro de los marcos gerenciales, ya sea tanto en la dirección al trazado de estrategias que proporcionen el alcance de los objetivos, en la organización en cuestión de coordinación de los recursos humanos con 
los recursos materiales, en la planificación de acciones a corto, mediano y largo plazo y en el control de la eficiencia por medición y comparación de los resultados con los objetivos propuestos.

El análisis de los estados financieros se caracteriza por ser una operación fundamentada en la reclasificación, recopilación, obtención y comparación de datos contables, operativos y financieros de una organización, que mediante la utilización de técnicas y herramientas adecuadas busca evaluar la posición financiera, el desarrollo y los resultados de la actividad empresarial en el presente y pasado para obtener las mejores estimaciones para el futuro (Nava, 2009).

En otras palabras, el análisis económico-financiero le confiere utilidad a la contabilidad para la toma de decisiones y este constituye su objetivo más importante.

El empleo de este método incluye métodos empíricos, económicos financieros, estadísticos y estratégicos, y a partir de una secuencia lógica de etapas y pasos, los procedimientos se vuelven detallados y fáciles de utilizar. También es una alternativa flexible, puesto que cada etapa emite su resultado pertinente y permite evaluarlos en cuanto el enriquecimiento analítico que aportan. Otro aspecto importante, que evidencia su evolución, es su cualidad explorable, es decir, que es aplicable a cualquier organización.

Con 4 fases de funcionamiento, y una serie de etapas dentro de cada una, enfatiza sus procesos en la investigación, preparación y selección de técnicas, análisis e interpretación y comunicación.

La Fase I, conocida como investigación preliminar, en la fase de identificación de los objetivos básicos, la selección del tipo de análisis y alcance de este, así como del equipo de analistas. En esta fase también se define el objeto de estudio, las razones de la entidad elegida, el período de duración del análisis y las expectativas que se esperan de este.

Cabe destacar que el análisis debe comprender la situación de la empresa, y que este puede ser parcial o total de acuerdo con las dimensiones que abarque.

Es imprescindible también en la fase I determinar las características de la empresa en cuanto a estructura organizativa, objeto social, misión y visión, competitividad para poder definir la situación actual en la que se encuentra y los posibles escenarios futuros.

Para la Fase II, la fase de preparación de toda la información recopilada y los recursos disponibles, se definen los métodos y técnicas para el análisis determinadas por expertos del tema a analizar.

Ya en la Fase III de análisis e interpretación de los estados financieros a partir de los métodos seleccionados, la interpretación de los resultados determinará las debilidades y 
fortalezas de la empresa, y según la retroalimentación obtenida, se revisan los resultados en vistas a su capacidad de cumplir con los objetivos establecidos. En caso de que no sea suficiente, se puede evaluar la posibilidad de aplicar otras técnicas adicionales. De la conclusión de esta fase se obtiene los principales aspectos positivos y negativos de la entidad, y un diagrama de causa -efecto que evalúa las causas de los problemas, y a partir de esto se trazan las acciones que contribuyen a su solución y mejoramiento de la empresa.

La Fase IV consiste en la comunicación a las partes interesadas de los resultados del análisis.

Un dato interesante sobre el análisis económico financiero es que centra una importante parte del proceso en la selección de los especialistas más experimentados y profesionales y también en la selección de los métodos y técnicas más ajustables a las características de la empresa y sus necesidades de análisis. En cada una de las selecciones se aplica el método de selección de expertos (para la selección de especialistas) que determina el coeficiente de competencia de los especialistas, y el método de consenso (para la selección de técnicas), que después de una extensa investigación de métodos de análisis, seleccionan aquellos que tienen un grado de aceptación mayor que el 85\% (Frías et al., 2008).

Para el análisis de la información económica financiera, las técnicas más utilizadas son los métodos horizontal, vertical y proyectado.

El método horizontal o dinámico según Murillo (2017), consiste en el estudio comparativo entre el estado financiero correspondiente a un año $\mathrm{X}$, con respecto al del año establecido como base.

Es decir, que se toma un año modelo como referencia y a partir de los resultados de este se evalúan las variaciones de la situación de la empresa y conocer su empeoramiento o mejoría.

Método de Análisis Vertical o Estático: Para comprobar si una empresa es equitativa en la distribución de los activos con respecto a la financiación de la que depende. Dentro del conjunto A, se comprueba claramente de qué forma está compuesto cada estado contable (Murillo, 2017).

El análisis vertical está basado en el análisis del comportamiento de las razones financieras que de acuerdo con Álvarez (2016), se clasifican en: razones de liquidez, miden la capacidad de pago a corto plazo de la empresa para saldar las obligaciones que vencen. Implica, por tanto, la habilidad para convertir activos en efectivo. Razones de cobertura, miden la capacidad de pago a largo plazo de la empresa para pagar las obligaciones que vencen. Miden el grado de protección a los acreedores e inversionistas. 
Razones de actividad, miden la efectividad con que la empresa está usando los activos empleados. Razones de rentabilidad. miden la capacidad de la empresa para generar.

El método de análisis proyectado o estimado se trata de analizar estados financieros preformas o presupuestos mediante la elaboración de un programa de previsión y administración financiera y de operación, y que se basa en experiencias anteriores y en la previsión de las condiciones futuras.

Otras técnicas de gran ayuda son el punto de equilibrio y el método gráfico. El punto de equilibrio es como explica Domínguez-Jiménez (2010), es aquel nivel de producción para el cual la empresa no registra ni beneficios ni pérdidas, sino que iguala los ingresos derivados de las ventas con los costes totales, tanto fijos como variables.

El método gráfico por su parte es más práctico y puede utilizarse en los tres métodos, pues convierte la información seleccionada de los estados financieros y las cifras relevantes de los análisis anteriores, a todo tipo de gráficos.

El análisis económico financiero es muy enriquecido en técnicas y procedimientos contables interpretativos, y a la vez enriquece el conocimiento de la empresa desde comportamientos controlados y acertados que beneficien el logro de las metas propuestas, ya sea productivas, de expansión de mercados, de diversificación o de reducción de costos.

Conocer el por qué la empresa está en la situación que se encuentra, sea buena o mala, es importante para así mismo poder proyectar soluciones o alternativas para enfrentar los problemas surgidos, o para idear estrategias encaminadas a aprovechar los aspectos positivos (Gerencie, 2020).

\section{Resultados}

\section{Estudio económico financiero}

Para la creación de la agencia de viajes receptiva especializada VitalTur, es necesario tener solvencia para trabajar y hacer frente a los problemas a los que se pueda enfrentar durante su funcionamiento. Desde el punto de vista económico, debe cumplir con tres requisitos fundamentales: capital desembolsado mínimo, una fianza de depósito bancario o aval para responder a obligaciones frente a consumidores y reembolsos, repatriación, compensaciones por quiebra y crisis y; por último, se debe de tener una póliza de seguros como garantía de solución a los problemas, que puedan presentarse, en la operación turística de la agencia.

El estudio económico financiero aportará los resultados finales para aprobar la creación de la agencia. Para ello se estimaron los gastos que incurrieron en la creación de la agencia de viajes, al igual que los gastos fijos, variables y de promoción de esta. 


\section{Conciencia}

\section{Tabla 1}

Gastos de creación de la agencia de viajes VitalTur

\begin{tabular}{lr}
\hline Concepto & Importe (\$) \\
\hline Capital Desembolsado mínimo & 800000.00 \\
Depósito de seguro & 100000.00 \\
Póliza de seguro & 80000.0 \\
Costos (trámites, licencias e impuestos) & 50000.00 \\
Otros gastos de apertura & 10000.00 \\
Inversión inicial & 1040000.00
\end{tabular}

Tabla 2

Gastos fijos y variables

\begin{tabular}{lr}
\hline \multicolumn{1}{c}{ Variables } & Importe (\$) \\
\hline \multicolumn{1}{c}{ Gastos Fijos } \\
\hline Equipamiento & 30000.00 \\
Materiales de oficina & 8000.00 \\
Total de Gastos Fijos & 38000.00 \\
\hline \multicolumn{1}{c}{ Gastos Variables (anual) } \\
\hline Alquiler del local & 3500.00 \\
Electricidad & 4000.00 \\
Teléfono & 2000.00 \\
Fax & 1000.00 \\
Internet & 1500.00 \\
Agua & 12000.00 \\
Nómina del personal (salarios totales) & 15000.00 \\
& \\
\hline
\end{tabular}




\section{Tabla 3}

Gastos en promoción e información (anual)

\begin{tabular}{lr}
\hline \multicolumn{1}{c}{ Variables } & Importe (\$) \\
\hline Logotipo & 2000.00 \\
Operación de relaciones públicas & 3000.00 \\
Gastos de información y suscripciones & 5000.00 \\
Plegables & 1000.00 \\
Brochures & 1500.00 \\
Total de gastos de promoción e información & 12500.00 \\
\hline
\end{tabular}

Se realizaron proyecciones en ventas para los primeros 5 años de funcionamiento de la agencia (este análisis se realiza a partir de la cotización realizada a los diferentes productos turísticos ofertados por la agencia de viajes) Se tuvieron en cuenta solo los precios de las habitaciones sencillas de cada producto, al ser las de mayor precio público.

\section{Tabla 4}

Proyecciones de ventas para los primeros 5 años

\begin{tabular}{|c|c|c|c|c|c|c|c|c|c|c|}
\hline Aspectos & & Año 1 & & Año 2 & & Año 3 & & Año 4 & & Año 5 \\
\hline Tour & 15 & 32970.00 & 20 & 43960.00 & 25 & 54950.00 & 30 & 65940.00 & 35 & 76930.00 \\
\hline Viñales & PAQ & & PAQ & & PAQ & & PAQ & & PAQ & \\
\hline Conociendo & 10 & 26350.00 & 15 & 39525.00 & 20 & 52700.00 & 25 & 65875.00 & 30 & 79050.00 \\
\hline mi Habana & PAQ & & PAQ & & PAQ & & PAQ & & PAQ & \\
\hline Maravillas & 15 & 20445.00 & 20 & 27260.00 & 25 & 34075.00 & 30 & 40890.00 & 35 & 456605.00 \\
\hline del Centro & PAQ & & PAQ & & PAQ & & PAQ & & PAQ & \\
\hline Total de & & $\$ 79765.00$ & & $\$ 110745.00$ & & $\$ 141725.00$ & & $\$ 172705.00$ & & $\$ 612585.00$ \\
\hline
\end{tabular}

Se realizó un flujo de caja, con el objetivo de determinar el saldo final en los primeros 5 años de la agencia. 


\section{Tabla 5}

Flujo de Caja

\begin{tabular}{lcccccc}
\hline Aspectos & Año 0 & Año 1 & Año 2 & Año 3 & Año 4 & Año 5 \\
\hline $\begin{array}{l}\text { Saldo } \\
\text { inicial }\end{array}$ & $\$ 1040000.00$ & $\$ 1002000.00$ & $\$ 1029265.00$ & $\$ 1081676.00$ & $\$ 1158585.00$ & $\$ 1259272.00$ \\
Entradas & & 79765.00 & 110745.00 & 141725.00 & 172705.00 & 612585.00 \\
de \\
efectivo
\end{tabular}

Nota: Se consideraron los gastos fijos y los gastos variables y de promoción.

Luego de realizado el estado de resultado, logrado a partir de estimaciones, se comprobó, que en los primeros cinco años se obtendrán beneficios netos.

\section{Tabla 6}

\section{Estado de resultado}

\begin{tabular}{lccccc}
\hline & Año 1 & Año 2 & Año 3 & Año 4 & Año 5 \\
\hline Ventas & 79765.00 & 110745.00 & 141725.00 & 172705.00 & 612585.00 \\
(-) Costo de Ventas & 25600.00 & 35000.00 & 37900.00 & 40320.00 & 45000.00 \\
Ventas Netas & 54165.00 & 75745.00 & 103.825 & 132385.00 & 564585.00 \\
$(-)$ Gastos de Operación & 3500.00 & 5560.00 & 7200.00 & 8300.00 & 10200.00 \\
$\begin{array}{l}\text { (-) Gastos de } \\
\text { administración }\end{array}$ & 15000.00 & 15000.00 & 15000.00 & 15000.00 & 15000.00 \\
Beneficio Neto & & & & & \\
& $\$ 35665.00$ & $\$ 55185.00$ & $\$ 81625.00$ & $\$ 109085.00$ & $\$ 539385.00$ \\
\hline
\end{tabular}




\section{Punto de Equilibrio}

El punto de equilibrio se calculó solo para el primer año. Para el análisis de este cálculo se tuvieron en cuenta todos los gastos fijos y variables que incurren en la agencia de viajes. Además, se tomó como referencia solo tres productos turísticos cotizada (circuitos, excursión y paquetes), con los cuales se puede realizar una estimación aproximada del precio del resto de los productos ofertados.

Se realizó un promedio entre el precio de estos productos, para de esta manera analizar la cantidad de personas que deben comprar los paquetes para que la agencia de viajes creada no obtenga pérdidas.

Los precios de venta de los productos son: \$2198.00; \$ 2635.00: \$ 1363.00; por lo que se llegó a un precio promedio de \$2065.00 aproximadamente, el cual se considera como precio de venta por persona, ya que cada paquete está diseñado para que lo pague una persona, independiente de la cantidad que se incluyan para su realización.

Punto de equilibrio $=$ Costos Fijos totales $/$ precio de venta por personas - costos variables por persona.

Punto de equilibrio $=\$ 38000.00 / \$ 2065.00-\$ 2000.00$

Punto de equilibrio $=\$ 38000.00 / 65$

Punto de Equilibrio = 584.6

Para poder vender el paquete con ganancia, se debe de vendar mínimo a 585 personas aproximadamente.

Costo variable total $=$ costo variable por persona $\mathrm{x}$ cantidad de personas

(Se considerarán 20 personas, ya que cada producto fue cotizado y diseñado para este mínimo de capacidad)

$$
\begin{aligned}
& \$ 57000.00=C V p \text { x } 20 \text { pax } \\
& C V p=\$ 40000.00 / 20 \text { pax } \\
& C V p=\$ 2000.00
\end{aligned}
$$

Periodo de Recuperación de Descuento 


\section{Tabla 7}

Periodo de recuperación de descuento

\begin{tabular}{|c|c|c|c|c|c|}
\hline & Año 0 & Año 1 & Año 2 & Año 3 & Año 4 \\
\hline FCN & $-\$ 1040000.00$ & $\$ 1029265.00$ & $\$ 1081676.00$ & $\$ 1158585.00$ & $\$ 1259272.00$ \\
\hline$K=10 \%$ & & 0.9091 & 0.8264 & 0.7513 & 0.6830 \\
\hline FCND & $-\$ 1040000.00$ & $\$ 935704.70$ & $\$ 893897.00$ & $\$ 870444.91$ & $\$ 860082.8$ \\
\hline Acumulado & $-\$ 1040000.00$ & $-\$ 104296.00$ & $\$ 789601.00$ & $\$ 1659645.00$ & $\$ 10259727.00$ \\
\hline
\end{tabular}

PRD $=1+($ Último Acumulado Negativo / FCND sig. Al último acumulado negativo * 365 días

$\mathrm{PRD}=1+(104296.00 / 893897.00 * 365$ días $)$

$\mathrm{PRD}=1+(0.12 * 365$ días $)$

PRD $1+44$ días

PRD = 1 años y 44 días aproximadamente

Diseño preliminar de los productos principales y servicios que se ofertarán

Un ejemplo de tour de naturaleza, que propone la agencia de viajes, es el Tour a Viñales: "Juntos en Viñales", en la provincia de Pinar del Río. Se tiene previsto para 20 personas como mínimo, estancia de 3 noches en el Hotel E. Central Viñales, con plan Americano Modificado (Media Pensión: MAP). Se prevé su realización en los días del 2 al 5 de abril. Como la entrada al hotel es por la tarde, se les propone a los clientes disfrutar de una cena especial el Día 1, donde podrán disfrutar de canciones del ayer, interpretadas por los músicos del hotel. En cuanto al itinerario, el Día 2, se realizará un tour por el Valle Viñales con visitas a la Cueva del Indio y al Palenque de los Cimarrones. El recorrido se hará en un minibús privado, con guías especializados y conocedores de la zona. La entrada a ambos lugares se contará en el precio final del paquete, de manera tal, que los clientes no se preocupen por ello. Como los adultos de la tercera edad prefieren no saltarse las comidas, el almuerzo se llevará a cabo entre una y otra visita, en el restaurante "Las 
tres hojas", que posee variedad de platos tradicionales cubanos. A las 5:00 pm se retornará al hotel y a las 7:00 pm se les ofrecerá la cena. El Día 3, se realizará un recorrido por la Casa del Veguero, donde disfrutarán de la demostración de la fabricación de puros y su degustación, si lo desean. Al mediodía, se les propicia la experiencia única en el Hotel "Los Jazmines", en el cual podrán disfrutar de unas bellísimas vistas y de unos platos exclusivos. A las 4:00 pm, retornarán al hotel. Al otro día a la hora prevista, se le garantiza el traslado al aeropuerto para abordar su vuelo de retorno.

Además, se poseen ofertas culturales y de esparcimiento, entre las cuales se destaca la propuesta: "Conociendo mi Habana", la cual cuanta con varias rutas turísticas asignadas por día. La misma tendrá una duración de cuatro noches y cinco días, propuesta para el mes de septiembre. En esta, se propone el alojamiento en el Hotel Ambos Mundos (plan $\mathrm{CP}$ ), pues se desea que los clientes puedan interactuar con la población de la zona y la cultura cubana, en general. Todos los clientes tendrán incluido en el paquete, el derecho a participar en las actividades para incentivar la salud física y mental en el parque "Los Capitanes Generales", en las cercanías. Entre estas, se concibieron: el taichí y ejercicios aeróbicos. En el Día 1, realizarán la visita al "Museo de los Capitanes Generales" y, posteriormente, disfrutarán del almuerzo en el restaurante "El Patio". Por la tarde, podrán asistir a una clase especial en la "Casa del Son", donde podrán bailar al compás de diferentes ritmos como: el son y el chachá. En el Día 2, se les propone un recorrido por el "Cristo Redentor" y un almuerzo en el Restaurante "La Divina Pastora", donde podrán deleitarse con la música en vivo y disfrutar de una gastronomía selecta. En el Día 3 es una ruta de degustación, se les propone una visita a la "Casa del Chocolate", donde recibirán una conferencia acerca del proceso de fabricación de los chocolates en la entidad y podrán deleitarse con un dulce, incluido en la oferta. Posteriormente se realizará un almuerzo en el bar-restaurante "El Floridita", donde se realizará además una degustación del famoso daiquirí, luego en la noche se efectuará una cena en el emblemática "Bodeguita del Medio". En el Día 4, se realizará una visita guiada al Museo de Ciencias Naturales y a los Museos de Bellas Artes, tanto el Nacional como el Internacional y, posteriormente, un almuerzo especial en el Restaurante "La Mina". En la noche para clausurar las rutas culturales por las calles habaneras, se realizará una visita al Gran teatro nacional Alicia Alonso para disfrutar de un espectáculo de la compañía del propio nombre.

Como último ejemplo, se hace referencia el Circuito "Maravillas del Centro", para 20 personas, el cual se puede llevar a cabo en el mes de octubre. En el mismo se propone el traslado de La Habana-Santi Spíritu- Cienfuegos. El día de llegada, se les hará un recorrido por la ciudad de Cienfuegos, incluyendo la visita al Museo Terry y terminando en el hermoso malecón. Además, podrán disfrutar de una maravillosa cena en el Restaurante "Covadonga", ubicado en el mismo malecón y con vistas espectaculares. Luego se realizará una excursión a Trinidad. Allí, acompañados de guías especializados, 
visitarán el Museo Romántico y el Valle de los Ingenios. También disfrutarán de un almuerzo en la Plaza Santa Ana. Al regresar a Cienfuegos, recorrerán la Plaza del Parque José Martí y culminara la travesía con una excursión al Nicho, donde podrán disfrutar de la maravillosa vegetación del lugar y baño en el río.

\section{Conclusiones}

- El informe realizado demuestra la veracidad de la hipótesis planteada para la elaboración de este, pues la agencia de viajes Vitaltur garantiza la satisfacción del mercado de la tercera edad para el destino Cuba por medio de la elaboración de ofertas atractivas con facilidades especiales para el segmento seleccionado.

- Queda demostrada la necesidad creciente del segmento adulto mayor de contar con servicios que promuevan la realización de actividades turísticas de manera segura y confortable.

- La comercialización de los paquetes turísticos de diferentes temáticas y que abarcan diferentes regiones del país garantizarán la sustentabilidad de la empresa por los próximos cinco años y la satisfacción del cliente sénior europeo.

- La creación de la agencia Vitaltur será sostenible en tanto se desarrolle una correcta comunicación con el cliente y se garantice una excelente relación calidadprecio que permitan brindar el servicio que merece este grupo especial.

\section{Referencias Bibliográficas}

Álvarez, L. N. (2016). Finanzas 1: Contabilidad, planeación y administración financiera.

Domínguez -Jiménez, J. S. (2010). Análisis Económico - Financiero. Escuela de Organización Industrial, Creative Commons Reconocimiento.

Frías Jiménez, R., González Arias, M., \& Cuétara Sánchez, L. (2008). Herramientas de apoyo a la solución de problemas no estructurados en empresas turísticas (Haspnet). Matanzas: Universidad de Matanzas.

Gerencie. (25 de enero de 2020). Análisis de estados financieros. Obtenido de https://wwwgerencie.com/análisis-de -estados-financieros.html.

Laborde, O. D. (2009). Elaboración del Plan de Negocio en la Agencia de Viajes Cubatur Villa Clara. Universidad Central "Marta Abreu" de las Villas. Trabajo de Diploma. Facultad de Ingeniería Industrial y Turismo.

Murillo, N. M. (2017). Análisis financiero de TR Hotel Jardín del Mar S.A en opción al Turismo. Facultat de Ciences Jurídques i Economiques. JAUME I. 
Nava, R. (2009). Análisis financiero: una herramienta clave para una gestión financiera eficiente. Revista Venezolana de Gerencia.

Nogueira-Rivera, D., Medina León, A., \& Hernández -Nariño, A. (2017). Análisis económico-financiero: talón de Aquiles de la organización. Caso de aplicación. Ingeniería Industrial.

Organización Mundial de la SALUD [OMS]. (2021). Envejecimiento y ciclo de vida. http://www.who.int/ageing/about/facts/es/

Organización Mundial del Turismo [OMT]. (28 de septiembre de 2016). World Tourism Organization. http://media.unwto.org/es/press-release/2016-09-29/

Pomeda, J. R. (2001). Gestión de Agencia de Viajes. Universidad de ExtremaduraInstituto Paulista de Ciencias de Administración.

\section{Ciencia Digital}




\section{Conciencia}

El artículo que se publica es de exclusiva responsabilidad de los autores y no necesariamente reflejan el pensamiento de la Revista Conciencia Digital.

\section{Ciencia \\ Ligital}

El artículo queda en propiedad de la revista y, por tanto, su publicación parcial y/o total en otro medio tiene que ser autorizado por el director de la Revista Conciencia Digital.
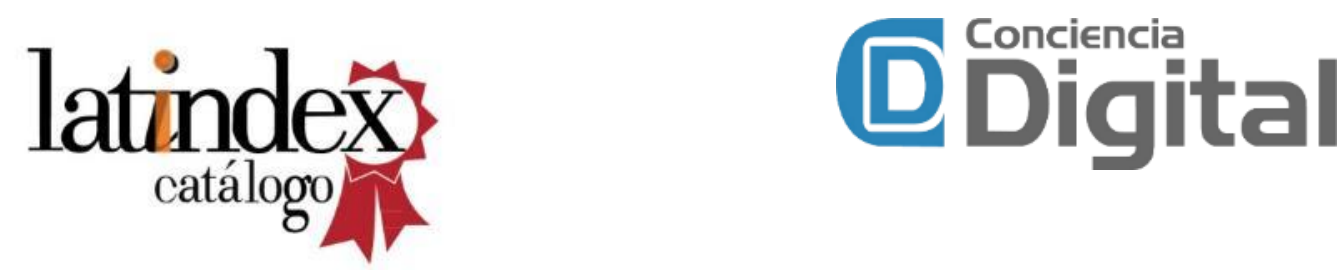

Indexaciones

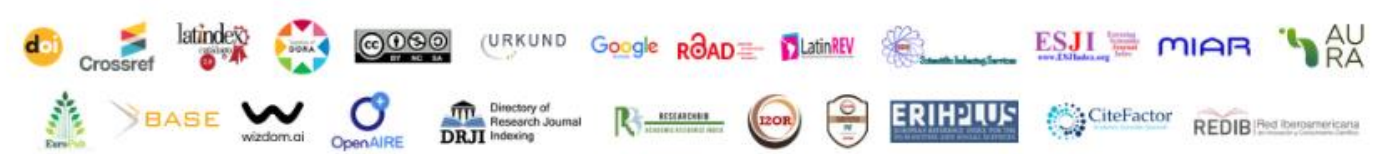

